\title{
Mammakarzinom
}

\section{Gedächtnis durch Zytokine beeinträchtigt?}

Bereits vor Beginn jeglicher Therapie inklusive der Operation sind bei Frauen mit Brustkrebs neurokognitive Defizite zu beobachten. Offensichtlich besteht eine Assoziation zu Zytokinen, wie eine aktuelle Studie zeigt. Darin wurden 174 postmenopausale Frauen mit vor Kurzem diagnostiziertem Brustkrebs, die noch nicht operiert wurden, sowie 88 altersgleiche Frauen ohne Brustkrebs einer umfassenden neuropsychologischen Evaluation mit Erhebung von kognitiver Funktion, Stimmung und Fatigue unterzogen. Darüber hinaus wurde die Rolle von zirkulierendem Interleukin-6 (IL-6), des Interleukin-1-Rezeptorantagonisten (IL-1RA) und des löslichen TNF-Rezeptors Typ 2 (sTNFR2) für die neurokognitive Leistungsfähigkeit untersucht.
Zur Prüfung der neurokognitiven Funktionen wurden die verbale Gedächtnisleistung, die Prozessgeschwindigkeit und die Exekutivfunktion erhoben. Interessanterweise war das verbale Gedächtnis die einzige kognitive Domäne, bei der es signifikante Unterschiede zwischen Patienten- und Kontrollgruppe gab $(\mathrm{p}=0,02)$.

Von den drei gemessenen Zytokinen war nur IL-1RA bei den Krebspatientinnen signifikant gegenüber der Kontrollgruppe erhöht (375 vs. 291 pg/ml; $\mathrm{p}=0,007)$; es erwies sich jedoch nicht als unabhängiger Parameter.

Nach Ausschluss möglicher Einflussfaktoren (Alter, Bildung, Herkunft, Stimmung, Fatigue, Body-Mass-Index und Komorbidität) konnten $6 \%$ der Gesamtvarianz der (verbalen) Gedächtnisleistung

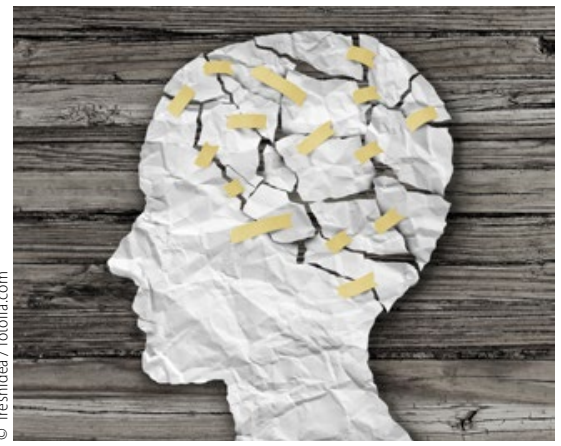

Möglicherweise richten Zytokine im Gehirn von Brustkrebspatientinnen Schaden an.

auf die Zytokine zurückgeführt werden $(p=0,01)$. Als Einzelfaktor war speziell ein höherer Spiegel des sTNFR2 mit einer schlechteren Gedächtnisleistung verbunden.

Brigitte Schalhorn

Patel SK et al. Inflammatory biomarkers, comorbidity, and neurocognition in women with newly diagnosed breast cancer. J Natl Cancer Inst. 2015; 107(8):djv131.

\section{Primärtherapie des fortgeschrittenen GIST Nilotinib nicht besser als Imatinib}

Nilotinib kann nicht für die Ersttherapie bei Patienten mit inoperablen und/oder metastasierten gastrointestinalen Stromatumoren (GIST) empfohlen werden. Das zeigte die Phase-III-Studie ENESTg1. In dieser wurden Patienten mit einem inoperablen und/oder metastasierten GIST täglich entweder mit 1-mal 400 mg Imatinib (KIT-Exon-9-Mutation: 2-mal 400 mg) oder 2-mal 400 mg Nilotinib behan- delt. Bei Progression war ein Wechsel in den anderen Studienarm vorgesehen.

In die Studie sollten 736 Patienten aufgenommen werden. Eine erste geplante Interimsanalyse (auswertbar: Daten von 397 Patienten) nach 75 Fällen mit Progression ergab, dass es im Nilotinib-Arm häufiger zur Progression gekommen war (24 vs.14\%; Hazard Ratio [HR] 2,03). Daraufhin wurde die Neuaufnahme weiterer

\section{kurz notiert}

\section{Steuerung der Zellteilung durch molekularen optischen Schalter}

Wirkstoffe wie z. B. Vincaalkaloide, die an den Mikrotubuli ansetzen, sind sehr wirkungsvolle Chemotherapeutika, führen aber auch zu schweren Nebenwirkungen, weil sie ihre zellschädigende Wirkung im ganzen Körper ausüben. Münchner Forschern ist es nun gelungen, in Colchicin, das ebenfalls die Mikrotubuli inhibiert, einen molekularen optischen Schalter einzubauen [Borowiak M et al. Cell. 2015;162(2):40311]. Das hat zur Folge, dass der Wirkstoff nur nach Bestrahlung mit blauem Licht aktiv ist. Damit wird es möglich, den Wirkstoff gezielt nur dort zu aktivieren, wo er erforderlich ist, nämlich in den Tumorzellen. Die Reaktion ist reversibel, d. h. durch Weglassen der Bestrahlung wird sie wieder abgeschaltet. Bezeichnet werden solche mit einem optischen Schalter ausgestattete Colchicin-Derivate als Photostatine. Im Zellversuch wurde die Funktionsfähigkeit der Photostatine bereits bewiesen: In Zellen, die mit blauem Licht bestrahlt wurden, hemmten sie die Zellteilung 250-mal stärker als in Zellen, die im Dunkeln gehalten wurden. Ein mögliches Einsatzgebiet für Photostatine könnten vor allem örtlich begrenzte Tumoren sein, die leicht mit einer Lichtquelle erreicht werden können, z. B. Retinoblastome.
Patienten gestoppt. In der endgültigen Analyse $(\mathrm{n}=644)$ war das progressionsfreie 2-Jahres-Überleben unter Imatinib mit 59,2\% höher als unter Nilotinib mit $51,6 \%$ (HR 1,47). Nach 24 Monaten überlebten im Imatinib-Arm mit 90,0\% mehr Patienten als unter Nilotinib mit 81,8\% (HR 1,85).

An Grad-3/4-Nebenwirkungen standen unter Imatinib Hypophosphatämie (6\%), Anämie (5\%), erhöhte Lipase $(5 \%)$ und Bauchschmerzen (4\%) und in der Nilotinib-Gruppe Anämie (6\%), erhöhte Lipasewerte (5\%), erhöhte SGPT (4\%) und Bauchschmerzen (3\%) im Vordergrund.

In der Subgruppenanalyse der Patienten mit KIT-Exon-9-Mutation waren das progressionsfreie Überleben und das Überleben nach 24 Monaten bei niedrigen Fallzahlen $(\mathrm{n}=50)$ unter Imatinib höher als unter Nilotinib. Bei Exon11-Mutation $(n=266)$ fanden sich bezüglich des progressionsfreien Überlebens keine Unterschiede, wohl aber in der Überlebensrate, die unter Imatinib mit $96,2 \%$ höher war als unter Nilotinib mit $87,5 \%$ (HR 3,00).

Brigitte Schalhorn

Blay JY et al. Nilotinib versus imatinib as first line therapy for patients with unresectable or metastatic gastrointestinal stroma tumors (ENESTg1): a randomised phase 3 trial. Lancet Oncol. 2015; 16(5):550-60. 